\title{
Head and neck
}

\section{squamous cell carcinoma survivorship care}

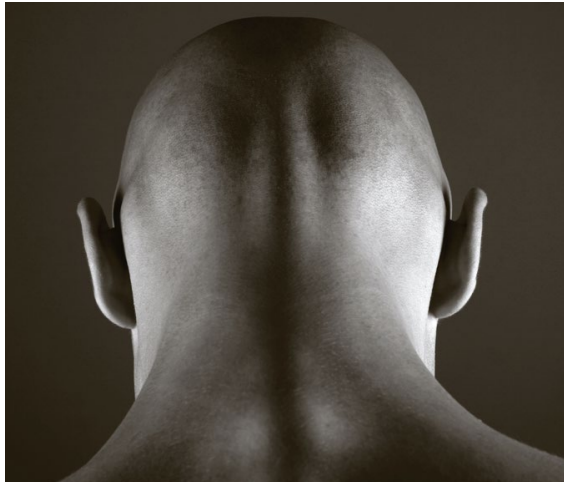

CPD

Joo Koh, Patrick Walsh, leta D'Costa, Osama Bhatti

\section{Background \\ Head and neck cancer is the seventh most commonly diagnosed cancer in Australia. More than $90 \%$ of these cancers are squamous cell carcinomas (SCCs). With advances in cancer therapies, survivorship care is becoming increasingly topical. In particular, general practitioners (GPs) will play an increasingly central part in the management of this unique cohort of patients.}

\section{Objective}

The aim of this article is to provide a succinct overview of long-term follow-up and surveillance for the head and neck SCC survivor.

\section{Discussion}

Treatment of head and neck SCC, particularly advanced cases, often requires a combination of surgery, radiotherapy and cytotoxic chemotherapy. As a result, the longterm follow-up care of head and neck SCC survivors is complex and has many facets including physical and mental treatment sequelae. GPs play a vital part in follow-up, particularly in disease surveillance and managing treatment complications.
HEAD AND NECK CANCER (HNC) is the seventh most commonly diagnosed cancer in Australia. The number of patients diagnosed with HNC in 2017 was double the number diagnosed in 1982 and is predicted to continue to increase. ${ }^{1} \mathrm{HNC}$ has a male predominance, with twice as many men diagnosed as women. ${ }^{1}$ With modern cancer treatments, it is estimated that $70 \%$ of patients with $\mathrm{HNC}$ will survive beyond five years, with many of these patients projected to become 'long-term survivors'. In 2012, there were 41,550 survivors of HNC in Australia. ${ }^{1}$

With advances in cancer therapies, survivorship care is becoming increasingly topical. The issues faced by survivors of HNC extend beyond the physical: a cancer diagnosis can have lasting psychosocial effects. ${ }^{2}$ As the treatment paradigm transitions from acute care to chronic disease management, general practitioners (GPs) will play an increasingly central part in the management of this unique cohort of patients. Some large Australian cancer centres are already trialling shared-care models involving oncologists and GPs. ${ }^{3}$

This article provides a guide on long-term follow-up and surveillance for the survivor of HNC.

\section{Overview of treatment of head and neck cancer}

More than $90 \%$ of head and neck malignancies are squamous cell carcinomas (SCCs) involving the mucosa of the oral cavity, pharynx and larynx. This article focuses on the management of mucosal head and neck SCCs. Other pathologies in the head and neck region include SCCs of the skin, salivary gland tumours, paranasal sinus tumours and skin malignancies including melanomas; these are less common and their management is covered elsewhere. ${ }^{4,5}$

In the case of mucosal head and neck SCCs, smoking and alcohol consumption are major risk factors, accounting for an estimated $75 \%$ of cases. ${ }^{6}$ High-risk strains of human papillomavirus (HPV) account for up to $70 \%$ of oropharyngeal SCCs. HPV-associated oropharyngeal SCCs have a better prognosis in the early stages, particularly for non-smokers, with five-year survival post-treatment approaching $90 \% .^{7}$

The treatment of head and neck SCC depends on the anatomical site, tumour stage and functional outcome. Early stage cancers (Stage I and II) are treated with a single modality - surgery or radiotherapy alone. Approximately $80-90 \%$ of patients with early-stage disease will be cured. Locally advanced cancers (Stage III and IV) require multimodality treatment - often a combination of surgery, radiotherapy and cytotoxic chemotherapy.

Although survivors of $\mathrm{HNC}$ are a heterogeneous group, there are some common themes in their follow-up care (Box 1), which are outlined in this article. 


\section{Early detection of disease recurrence}

Head and neck SCCs rarely metastasise distantly. The majority of disease recurrences are localised to the head and neck, ${ }^{8}$ with the highest risk period in the first five years post-treatment. The American Society of Clinical Oncology guideline recommends that a focused head and neck history and physical examination be performed every three months in the first year post-treatment, every 2-6 months in the second year, every 4-8 months in years 3-5 and annually after the fifth year. ${ }^{6}$ In Australia, patients commonly will have scheduled reviews with their surgeon and oncologist during this time. However, it is still important for GPs to be able to recognise the clinical features of cancer recurrence (Table 1 ), and refer to a head and neck specialist for further assessment.

\section{Treatment complications}

\section{Acute radiation toxicities}

Radiation mucositis and pharyngitis are common side effects of head and neck radiotherapy that result in odynophagia. Symptoms typically begin 2-3 weeks after the start of radiotherapy and increase gradually in severity, peaking at 2-4 weeks after the completion of radiation. Many patients have severe mucositis/pharyngitis at the end of treatment and require enteral feeding for nutritional support. Acute radiation toxicities should be managed by the radiation oncology team, who review patients on a weekly basis during treatment. Common analgesic regimens for mucositis/pharyngitis include topical analgesia, such as lignocaine viscus and cocaine mouthwash, as well as opioid analgesia. Oral thrush is common, and this is treated with bicarbonate mouthwashes

\footnotetext{
Box 1. Goals of follow-up care

- Early detection of disease recurrence

- Detection and management of treatment complications

- Screening for and prevention of second primary malignancies

- Psychosocial care
}

and nilstat drops. Severe odynophagia that does not improve 6-8 weeks post-radiotherapy is of concern, and a prompt referral to the radiation oncology team is warranted.

\section{Dysphagia}

Dysphagia is a debilitating symptom for many patients with head and neck SCC.

Dysphagia can be present pre-treatment as a direct effect of the tumour. All treatment modalities, particularly surgery and radiotherapy, can lead to significant dysphagia. Neuropathies affecting swallowing can be a late complication of radiotherapy. Besides patient-reported symptoms of difficulty swallowing, other potential issues may include blockage of food boluses, weight loss and recurrent pneumonias from aspiration. Aside from surgical management for anatomical causes of dysphagia, the mainstay of treatment is centred on behaviour-focused swallowing interventions by speech pathologists. ${ }^{9}$

\section{Lymphoedema}

Lymphoedema is chronic swelling of the skin and soft tissue due to disruption of lymphatic channels from surgery or radiotherapy. It can be external (eg face, neck) or internal (eg larynx, pharynx, oral cavity) and affects up to $75 \%$ of survivors. ${ }^{10}$ Patients often complain of neck tightness or stiffness prior to the development of overt swelling. Treatment is complete decongestive therapy performed by a physiotherapist and comprises manual lymphatic drainage, compression and exercise programs. ${ }^{11}$ Early referral to a lymphoedema physiotherapist can improve pain, range of movement and progression.

\section{Hypothyroidism}

Patients who have had radiotherapy to the neck are at risk of hypothyroidism. This can occur from four weeks to 10 years after treatment, affecting up to $20 \%$ of patients at five years. ${ }^{12}$ Patients should have thyroid stimulating hormone measured every 6-12 months. Treatment of radiationinduced hypothyroidism is with thyroxine replacement.

\section{Hearing loss}

Patients can have an acute or progressive decline in hearing after treatment. Sensorineural hearing loss can result from ototoxic chemotherapy agents. Patients who have surgery or radiation to the nasopharynx, oropharynx or larynx can develop eustachian tube dysfunction. This can lead to middle ear effusion, resulting in conductive hearing loss. Patients with suspected hearing loss should be referred for formal audiology assessment. Long-standing middle ear effusions can be referred for surgical

\section{Table 1. Signs and symptoms of disease recurrence}

\begin{tabular}{|c|c|}
\hline History & $\begin{array}{l}\text { - New-onset pain with/without associated self-detected lesion/lump } \\
\text { - Functional changes such as odynophagia, dysphagia, voice change, nasal } \\
\text { obstruction } \\
\text { - Loss of appetite with/without change in weight } \\
\text { - New-onset bleeding - epistaxis or haemoptysis }\end{array}$ \\
\hline Examination & $\begin{array}{l}\text { - Visible or palpable oral cavity lesions or ulcers } \\
\text { - New-onset skin lesions } \\
\text { - Palpable cervical lumps } \\
\text { - New-onset cranial nerve abnormalities: } \\
\text { - facial nerve palsy } \\
\text { - altered sensation } \\
\text { - hypoglossal palsy }\end{array}$ \\
\hline Red flags & $\begin{array}{l}\text { - Rapidly growing new lesions/lumps } \\
\text { - New-onset ear pain with no apparent ear pathology on assessment - } \\
\text { potentially referred pain from oropharyngeal malignancies } \\
\text { - New symptoms and signs in patients who continue to smoke }\end{array}$ \\
\hline
\end{tabular}


management with middle ear ventilation tubes, whereas patients with significant sensorineural hearing loss should be referred for hearing aids.

\section{Psychosocial care}

The diagnosis and treatment of head and neck SCC is confronting and can lead to long-term psychosocial issues including depression and anxiety. The disfiguring effects of treatment and concerns of disease recurrence can often exacerbate these feelings. Cancer Council Australia has an excellent guideline on psychosocial care of patients with cancer, which is available for GPs. ${ }^{13}$ Cancer Council Australia also has a telephone support line (13 11 20), where patients can seek information and support from qualified cancer nurses. Further referral to a clinical psychologist may be appropriate. Additionally, excellent resources are available online from the Australian Cancer Survivorship Centre to support GPs to provide optimal survivorship care.

\section{Second malignancy}

One-quarter of survivors of head and neck SCC will develop a second primary malignancy. ${ }^{2}$ Up to $30 \%$ of deaths in primary malignancy survivors are due to a second unrelated cancer; this is three times the number of deaths from distant metastases. ${ }^{8,14}$ The most common primary sites are the oesophagus, head and neck, and lung. ${ }^{15,16}$ A Queensland study

\section{Box 2. The general practice visit for the survivor of head and neck cancer}

1. Assessment of symptoms or signs of disease recurrence

2. Assessment for treatment complications:

a. annual thyroid stimulating hormone check

b. psychosocial health assessment

c. Iymphoedema assessment and referral if required

d. dysphagia assessment - new or progressive dysphagia can be a sign of recurrence and warrants a specialist referral

3. Discussion of preventive health:

a. smoking cessation

b. reducing alcohol intake showed that men previously diagnosed with HNC were 10 times more likely to develop oesophageal cancer than the general population. ${ }^{16}$ Radiation-induced malignancies are uncommon and do not occur until $>10$ years post-treatment.

There are no current consensus guidelines on secondary malignancy screening in this group. Routine gastroscopies or imaging are not required, and investigations should be guided by symptoms.

Ongoing smoking and excess alcohol consumption have been identified as major risk factors for secondary cancers. ${ }^{17}$ Smoking cessation is the most important intervention for this patient group.

\section{Conclusion}

With developments in treatment modalities and increasing survivorship of HNC, long-term follow-up and care for patients is increasingly focused on quality and function of life. As the primary carer, the GP plays an essential part in both disease surveillance and management of treatment complications (Box 2).

\section{Authors}

Joo Koh MBBS, Postgraduate Diploma of Surgical Anatomy, ENT Service Registrar, Western Health, Vic. joohoekoh@gmail.com

Patrick Walsh MBBS, FRACS (OHNS), ENT Surgeon, Western Health, Vic; Chair of Head and Neck Multidisciplinary Team, Western Health, Vic leta D'Costa MBBS, MRCP, FRANZCR, MA Radiation Oncologist, Peter MacCallum Cancer Centre, Vic; Member of Head and Neck Multidisciplinary Team, Western Health, Vic Osama Bhatti MBBS, FRACGP, General Practitioner, Monash University Health Services, Vic

Competing interests: None.

Funding: None.

Provenance and peer review: Not commissioned, externally peer reviewed.

\section{References}

1. Australian Institute of Health and Welfare. Head and neck cancer in Australia. Cancer series no. 83. Cat. no. CAN 80. Canberra: AIHW, 2014.

2. Nekhlyudov L, Lacchetti C, Davis NB, et al. Head and neck cancer survivorship care guideline: American Society of Clinical Oncology Clinical Practice Guideline Endorsement of the American Cancer Society Guideline. J Clin Oncol 2017;35(14):1606-21. doi: 10.1200/ JCO.2016.71.8478.

3. Evans J, Nolte L, Piper A, Simkiss L, Whitfield K, Jefford M. A clinical placement program for primary care professionals at a comprehensive cancer centre. Aust Fam Physician 2016;45:606-10.
4. Margolis SA. Pigmented skin lesions. Aust J Gen Pract 2019;48(6):343. doi: 10.31128/AJGP-0619-1234e.

5. Sinclair R, Meah N, Arasu A. Skin checks in primary care. Aust J Gen Pract 2019;48(9):614-19. doi: 10.31128/AJGP-03-19-4887.

6. Cohen EE, LaMonte SJ, Erb NL, et al. American Cancer Society Head and Neck Cancer Survivorship Care Guideline. CA Cancer J Clin 2016;66(3):203-39. doi: 10.3322/caac.21343.

7. Lin BM, Wang H, D'Souza G, et al. Long-term prognosis and risk factors among patients with HPV-associated oropharyngeal squamous cell carcinoma. Cancer 2013;119(19):3462-71. doi: 10.1002/cncr.28250.

8. Oksuz DC, Prestwich RJ, Carey B, et al. Recurrence patterns of locally advanced head and neck squamous cell carcinoma after 3D conformal (chemo)-radiotherapy. Radiat Oncol 2011;6:54 doi: 10.1186/1748-717X-6-54.

9. Greco E, Simic T, Ringash J, Tomlinson G, Inamoto $Y$, Martino R. Dysphagia treatment for patients with head and neck cancer undergoing radiation therapy: A meta-analysis review. Int J Radiat Oncol Biol Phys 2018;101(2):421-44. doi: 10.1016/j.ijrobp.2018.01.097.

10. Deng J, Ridner SH, Dietrich MS, et al. Prevalence of secondary lymphedema in patients with head and neck cancer. J Pain Symptom Manage 2012;43(2):244-52. doi: 10.1016/j. jpainsymman.2011.03.019.

11. Smith BG, Lewin JS. The role of lymphedema management in head and neck cancer. Curr Opin Otolaryngol Head Neck Surg 2010;18(3):153-58. doi: 10.1097/MO0.0b013e32833aac21.

12. Tell R, Lundell G, Nilsson B, Sjödin H, Lewin F, Lewensohn R. Long-term incidence of hypothyroidism after radiotherapy in patients with head-and-neck cancer. Int J Radiat Oncol Biol Phys 2004;60(2):395-400. doi: 10.1016/j. ijrobp.2004.03.020.

13. National Breast Cancer Centre and National Cancer Control Initiative. Clinical practice guidelines for the psychosocial care of adults with cancer: A summary guide for health professionals. Camperdown, NSW: National Breast Cancer Centre, 2005.

14. Baxi SS, Pinheiro LC, Patil SM, Pfister DG, Oeffinger KC, Elkin EB. Causes of death in longterm survivors of head and neck cancer. Cancer 2014;120(10):1507-13. doi: 10.1002/cncr.28588.

15. Morris LG, Sikora AG, Patel SG, Hayes RB, Ganly I. Second primary cancers after an index head and neck cancer: Subsite-specific trends in the era of human papillomavirus-associated oropharyngeal cancer. J Clin Oncol 2011;29(6):739-46. doi: 10.1200/JCO.2010.31.8311.

16. Youlden DR, Baade PD. The relative risk of second primary cancers in Queensland, Australia: A retrospective cohort study. BMC Cancer 2011;11:83. doi: 10.1186/1471-2407-11-83.

17. Maasland DH, van den Brandt PA, Kremer B, Goldbohm RA, Schouten LJ. Alcohol consumption, cigarette smoking and the risk of subtypes of head-neck cancer: Results from the Netherlands Cohort Study. BMC Cancer 2014;14:187. doi: 10.1186/1471-2407-14-187. 